\title{
FUSÃO DE DADOS BASEADA NO FILTRO DE KALMAN PARA ESTIMAÇÃO DO NÍVEL DA INTERFACE ÓLEO/ÁGUA EM PROCESSOS DE SEPARAÇÃO
}

\author{
LUCAS B. OLIVEIRA, OSCAR A. Z. SOTOMAYOR.
}

\author{
Departamento de Engenharia Elétrica, Centro de Ciências Exatas e Tecnologia, Universidade Federal de \\ Sergipe \\ Av. Marechal Rondon, s/n Jardim Rosa Elze, 49100-000, São Cristóvão, Sergipe. \\ E-mails: lucas_barros88@hotmail.com, oscars@ufs.br
}

\begin{abstract}
The success of the oil primary processing depends on the optimal performance of the separation process. Thus, the monitoring and control of the levels of the interfaces in the separator vessel, such as the level of the oil/water interface, are of utmost importance. The aim of this work is the development and implementation of an algorithm capable of generating an online measurement of oil/water interface level of a separation process, by the using Kalman filters and data fusion concepts, to improve the detection, certainty and reliability of data. Using the estimated signal rather than the signal generated by the physical sensors is expected to increase the efficiency of the separation process, and thus the performance of the oil production process.
\end{abstract}

Keywords_ _ Level sensors, oil/water interface, Kalman filter, data fusion, separation process.

Resumo - O sucesso do processamento primário de petróleo depende do ótimo desempenho do processo de separação. Assim, o monitoramento e controle dos níveis das interfaces no vaso separador, a exemplo do nível da interface óleo/água, são de extrema importância. O objetivo principal do presente trabalho consiste no desenvolvimento e implementação de um algoritmo capaz de gerar uma estimação on-line do nível da interface óleo/água de um processo de separação, por meio da utilização de filtros de Kalman e conceitos de fusão de dados, a fim de melhorar a detecção, certeza e confiabilidade dos dados. Com o uso do sinal estimado, ao invés do sinal gerado pelos sensores físicos, espera-se aumentar a eficiência do processo de separação e, portanto, o desempenho do processo de produção de petróleo.

Palavras-chave— Sensores de nível, interface óleo/água, filtro de Kalman, fusão de dados, processo de separação.

\section{Introdução}

Atualmente, a separação adequada de múltiplas fases vem sendo um grande desafio para as companhias petrolíferas, em especial a do óleo bruto. Uma grande parte dos separadores existentes, hoje em dia, é baseada no princípio da gravidade, onde na parte superior do mesmo encontra-se o gás, na parte inferior água e sólidos diversos, e entre o gás e a água encontra-se o óleo. Assim, para otimizar o processo de separação, é critica a necessidade do monitoramento e controle dos níveis e interfaces gás/óleo, óleo/água e água/areia.

São muitas as dificuldades de encontrar sistemas adequados para a medição dos níveis de múltiplas fases. Isto se deve a necessidade de alta confiabilidade, a grande quantidade de fluidos, a ambientes hostis e questões de segurança. A maioria dos sistemas disponíveis no mercado, hoje, é baseada em técnicas de ultrassom, radiação gama, capacitância ou deslocamento. Porém, todos esses possuem alguns inconvenientes

O maior desafio existente é controlar a saída de água dos separadores a fim de manter o nível da interface óleo/água numa posição desejada. Para isso é proposta a criação de um instrumento barato e eficiente para o monitoramento e operação dos separados de óleo. Este instrumento é baseado na fusão de dados de múltiplos sensores. Esta combinação de dados dos sensores é feita por meio da utilização de filtros de Kalman para estimação ótima dos níveis das interfaces, possibilitando que o controle destes níveis seja realizado de forma mais eficiente.

\section{Processo de Separação de Petróleo}

\subsection{Descrição do Processo}

O processo de separação consiste, em linhas gerais, em separar o fluido multifásico extraído dos poços, composto principalmente de água, óleo e gás, em suas diferentes fases. Nas unidades de produção de petróleo offshore, estas operações são realizadas na unidade de separação. No presente estudo, a unidade de separação é composta de um separador trifásico em série com uma bateria de hidrociclones, conforme mostrado na Figura 1.

O separador de produção trifásico possui formato cilíndrico horizontal e é composto por duas seções denominadas câmara de separação e câmara de óleo, ambas separadas por um vertedouro. A força gravitacional promove a segregação das fases na câmara de separação, formando uma fase rica em água (fase aquosa), uma fase rica em óleo (fase oleosa) e uma interface entre ambas as fases. A fase oleosa transborda sobre o vertedouro para a câmara 
de óleo, sendo em seguida transferida até os tratadores, que retiram sais e resíduos da água. O gás, já separado do óleo e da água, é canalizado para compressores sendo, então, direcionado a uma planta de tratamento, onde a umidade é retirada. A fase aquosa é encaminhada à unidade de tratamento, neste caso os hidrociclones, para redução de resíduos de óleo nos padrões especificados.

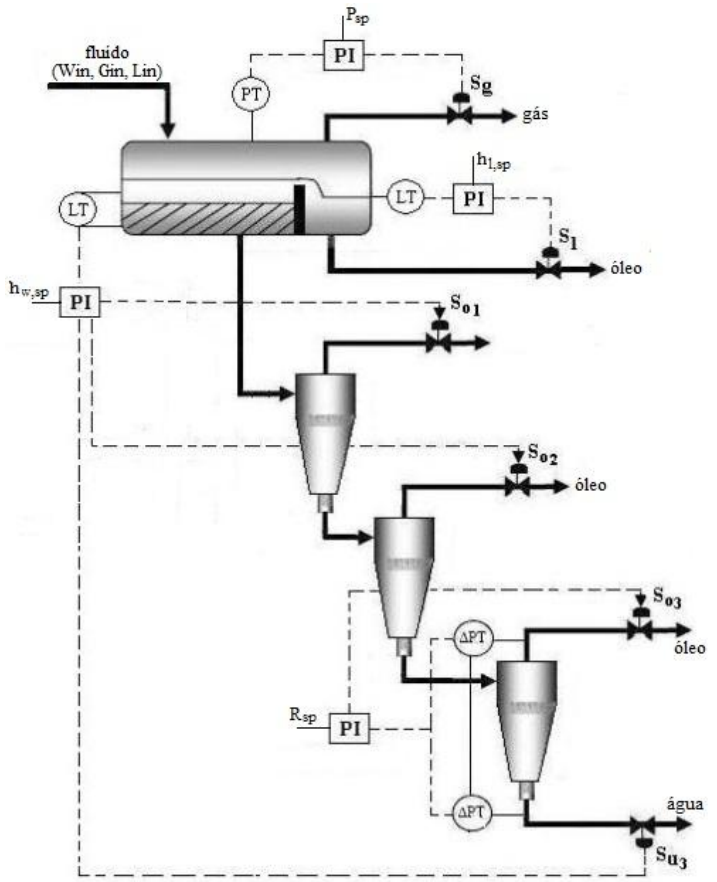

Figura 1. Unidade de Separação Trifásica (Adaptado de Silveira, 2006).

O simulador da unidade de separação utilizado neste trabalho foi implementado na plataforma Simulink/Matlab®, e é baseado em modelos fenomenológicos disponíveis na literatura. Para maiores detalhes vide Figueiredo (2009).

\subsection{Controle do Processo}

A unidade de separação opera sob um sistema de controle preditivo (MPC) atuando sobre uma camada inferior de controle regulatório, composto de quatro controladores PI (proporcional-integrativo).

Na camada inferior, o controle da pressão no separador $(P)$, o nível da fase oleosa na câmera de óleo $\left(h_{l}\right)$ e o nível da interface água/óleo na câmera de separação $\left(h_{w}\right)$ é realizada pela manipulação das aberturas da válvula de gás $\left(S_{g}\right)$, válvula de óleo $\left(S_{l}\right)$ e válvula de água $\left(S_{u 3}\right)$, respectivamente. Além disso, inclui-se um controle de razão para o último hidrociclone, utilizando um controlador PI para manter constante a relação entre as quedas de pressão da corrente de topo e de fundo $(R)$ pela manipulação da abertura da válvula de topo $\left(S_{03}\right)$, garantindo, desta forma, uma melhor eficiência de separação.

O controlador MPC manipula os setpoints dos controladores PI, enviando para estes o melhor setpoint possível para estabilizar o sistema, de forma a atenuar as perturbações que possam prejudicar o processo de produção. Assim, as variáveis manipuladas e controladas do MPC são os setpoints e as variáveis controladas dos PI's, respectivamente (Santos e Sotomayor, 2012).

\section{Fusão de Dados}

\subsection{Introdução}

O conceito de fusão de dados foi introduzido no início dos anos 70, em pesquisas médicas destinadas à produção de diagnósticos via métodos computacionais, assim como no campo militar, em estudos para identificação de alvos (aéreos, terrestres e aquáticos). Desde então, o problema da fusão de dados esteve relacionado à necessidade de se combinar dados precisos (natureza binária) e imprecisos (nebulosos), que apresentam diversos graus de incerteza (Filho et al, 2007).

Geralmente, a utilização da fusão de dados traz diversas melhorias. Essas vantagens envolvem principalmente melhorias na autenticidade ou na disponibilidade dos dados. Exemplos do primeiro são a melhor detecção, certeza e confiabilidade, bem como a redução na ambiguidade dos dados, enquanto a extensão da cobertura espacial e temporal pertence a esta última categoria de benefícios (Khaleghi, 2013). A aplicação de fusão de dados se estende por um vasto espectro, incluindo monitoramento ambiental, detecção e rastreamento automático de alvos, vigilância de batalhas, sensoriamento remoto, percepção global, etc. (Nazar, 2009).

\subsection{Filtro de Kalman Utilizando Fusão de Dados}

\subsubsection{O Filtro de Kalman}

Um dos métodos mais utilizados para fusão de dados e estimativas ótimas é o filtro de Kalman, onde é comumente realizada a combinação de informações, proveniente de dados quantitativos, baseando-se em algoritmos que atribuem ponderações para as informações disponíveis.

O filtro de Kalman é baseado em duas etapas: predição e atualização. A primeira etapa é responsável previsão do estado, já a segunda, é responsável pela correção dessa previsão com medições adquiridas do processo. Estas duas etapas são resumidas nas seguintes equações:

$$
\begin{gathered}
z_{k}=y_{k}-\mu_{v}-H_{k} \bar{x}_{k} \\
\hat{x}_{k}=\bar{x}_{k}+K_{k} z_{k} \\
\bar{x}_{k+1}=\emptyset_{k} \hat{x}_{k}+\mu_{w} \\
K_{k}=\bar{P}_{k} H_{k}^{T}\left[H_{k} \bar{P}_{k} H_{k}^{T}+R_{k}\right]^{-1} \\
\hat{P}_{k}=\left[I-K_{k} H_{k}\right] \bar{P}_{k} \\
\bar{P}_{k+1}=\emptyset_{k} \hat{P}_{k} \emptyset_{k}^{T}+Q_{k}
\end{gathered}
$$


onde,

- $z_{k} \quad$ - Inovação no instante $k$

- $y_{k} \quad$ - Medição no instante $k$

- $\mu_{v} \quad$ - Média do ruído das medições

- $H_{k}$ - Matriz de medições

- $\bar{x}_{k}$ - Estado

- $\hat{x}_{k}$ - Estimativa do estado

- $K_{k}$ - Ganho de Kalman

- $\bar{x}_{k+1}$ - Predição do estado

- $\emptyset_{k} \quad$ - Matriz de transição

- $\mu_{w}$ - Deslocamento do ruído do modelo

- $\bar{P}_{k}$ - Covariância

- $R_{k}$ - Matriz de covariância do ruído

- $\widehat{P}_{k}$ - Covariância estimada

- $\bar{P}_{k+1}$ - Predição da covariância

- $Q_{k} \quad$ - Matriz de covariância do modelo

\subsubsection{Métodos para Fusão de Dados Baseados em} Filtro de Kalman

Para fusão de dados, neste trabalho, são adotados dois tipos de métodos baseados em filtro de Kalman, a fusão de estimativas e a fusão de medições.

Por simplicidade, foi assumido que a taxa de amostragem dos sensores são idênticas e a dinâmica do alvo é representada por

$$
x_{k+1}=\phi_{k} x_{k}+\Gamma_{k} w_{k}
$$

onde $x_{k}$ é o vetor de estado no tempo $k$, e o estado do ruído $w_{k}$, tal que

$$
E\left[w_{k}\right]=0 ; \quad E\left[w_{k} w_{l}^{T}\right]=Q_{k} \delta_{k l}
$$

As medidas correspondentes para dois sensores são

$$
y_{k}^{m}=H_{k}^{m} x_{k}+v_{k}^{m}, \quad m=1,2
$$

onde $y_{k}^{m}$ é a medição do sensor $m$ no tempo $k$ e as sequencias de ruídos das medições $v_{k}^{m}$ são média zero, brancos, com covariância $R_{k}^{m}$, e independentes entre si, isto é,

$$
\begin{gathered}
E\left[v_{k}^{m}\right]=0 ; \quad E\left[v_{k}^{m} v_{k}^{m T}\right]=R_{k}^{m} \delta_{k} \\
E\left[v_{k}^{1} v_{l}^{2 T}\right]=E\left[v_{k}^{2} v_{l}^{1 T}\right]=0
\end{gathered}
$$

\section{a) Método de Fusão de Medições}

Existem essencialmente duas técnicas para a fusão de medições. A primeira simplesmente funde as medições em um vetor de observação aumentado e o segundo combina as medições utilizando estimativas por mínimos quadrados (Harris et al, 1998).

$\mathrm{Na}$ primeira técnica de fusão de medições, os vetores de medições $y_{k}^{1}$ e $y_{k}^{2}$ de dois (ou mais) sensores são fundidos em um novo vetor de medições aumentado dado por

$$
y_{k}=\left[\left(y_{k}^{1}\right)^{T}\left(y_{k}^{2}\right)^{T}\right]^{T}
$$

Denotando,

$$
\begin{aligned}
H_{k} & =\left[\left(H_{k}^{1}\right)^{T}\left(H_{k}^{2}\right)^{T}\right]^{T} \\
v_{k} & =\left[\left(v_{k}^{1}\right)^{T}\left(v_{k}^{2}\right)^{T}\right]^{T}
\end{aligned}
$$

então, das equações (9) e (12), uma nova equação de medições é dada por

$$
y_{k}=H_{k} x_{k}+v_{k}
$$

Com base no pressuposto de independência estatística dos dois sensores, a matriz de covariância $R_{k}$ para o ruído da medição fundido $v_{k}$ é definida como

$$
R_{k}=\left(\begin{array}{cc}
R_{k}^{1} & 0 \\
0 & R_{k}^{2}
\end{array}\right)
$$

Então, a estimativa, $x_{k \mid k}$, do vetor de estados pode ser determinada pelo filtro de Kalman tradicional por meio das equações (7) e (12).

Outra abordagem para a fusão de medições é ponderar as medições individuais de cada um dos sensores e, em seguida, utilizar as medições fundidas por um filtro de Kalman para obter uma estimativa do vetor de estados (Willner, 1976). Uma vez que o ruído das medições é independente para os sensores 1 e 2, a equação para fundir os vetores das medições $y_{k}^{1}$ e $y_{k}^{2}$, na forma recursiva, para uma estimativa média mínima quadrática é dada por

$y_{k}=y_{k}^{1}+R_{k}^{1}\left(R_{k}^{1}+R_{k}^{2}\right)^{-1}\left(y_{k}^{2}-y_{k}^{1}\right)$

onde $R_{k}^{m}$ é a matriz de covariância do vetor de medições do sensor $m$ definida por (10). Então, a matriz de covariância da medição fundida $y_{k}$ pode ser derivada por

$$
R_{k}=\left[\left(R_{k}^{1}\right)^{-1}+\left(R_{k}^{2}\right)^{-1}\right]^{-1}
$$


Estas medições fundidas podem então ser utilizadas para obter a estimativa do $x_{k \mid k}$ por um filtro de Kalman tradicional.

Na Figura 2, pode ser visto o algoritmo de fusão de dados por meio da fusão de medições.

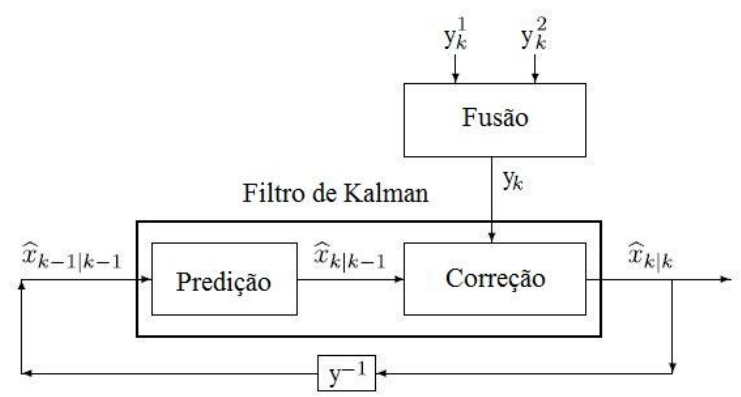

Figura 2. Processo de fusão de medições (Gao \&Harris, 2002).

\section{b) Método de Fusão de Estimativas}

O método de fusão de estimativa é um pouco diferente dos propostos anteriormente. Isso porque, ao contrário dos outros, a fusão é feita por último. Inicialmente são realizadas as estimativas utilizando cada uma das medições e, em seguida, estas estimativas são fundidas a fim de fornecer outra estimativa mais precisa e próxima do valor esperado. As estimativas podem ser realizadas por um filtro de Kalman tradicional ou suas derivações. Porém, para aplicar a fusão, são necessárias as equações seguintes.

$$
\begin{gathered}
\hat{x}_{k}=\bar{x}_{k}+\frac{1}{N_{0}} \sum_{i=1}^{N_{0}} K_{k} z_{k} \\
\bar{x}_{k+1}=\emptyset_{k} \hat{x}_{k} \\
K_{k}^{i}=\bar{P}_{k} H_{k}^{i^{T}}\left[H_{k}^{i} \bar{P}_{k} K_{k}^{i}+R_{k}^{i}\right]^{-1} \\
\widehat{P}_{k}=\left[I-\frac{1}{N_{0}} \sum_{i=1}^{N_{0}} K_{k}^{i} H_{k}^{i}\right] \bar{P}_{k} \\
\bar{P}_{k+1}=\emptyset_{k} \widehat{P}_{k} \emptyset_{k}^{T}+Q_{k}
\end{gathered}
$$

Onde as equações (19), (20), (21), (22) e (23), correspondem, respectivamente, a estimativa, predição, ganho de Kalman, covariância estimada e predição da covariância.

Na Figura 3, pode visto o algoritmo de fusão de dados por meio da fusão de estimativas.

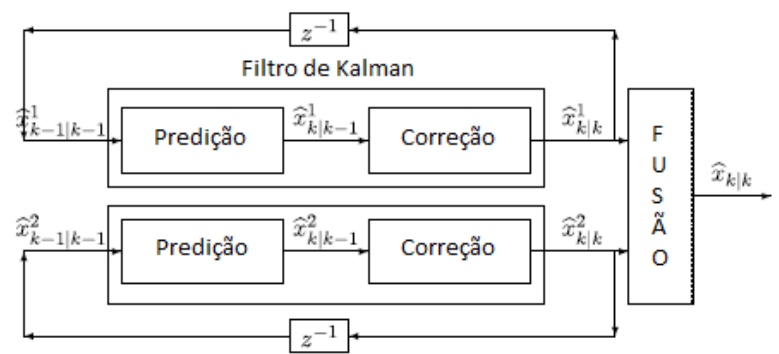

Figura 3. Processo de fusão de estimativas (Gao \&Harris, 2002).

\section{Aplicação da Fusão de Dados Baseada no Filtro de Kalman}

Um modelo em espaço de estado discreto do processo de separação, utilizado para implementação do filtro de Kalman, foi obtido por identificação em malha fechada através de métodos por subespaços:

$$
\begin{aligned}
& x((k+1) T)=\boldsymbol{A}_{d} x(k T)+\boldsymbol{B}_{d} u(k T) \\
& y(k T)=\boldsymbol{C}_{d^{x}} x(k T)
\end{aligned}
$$

Para aplicação da metodologia, no simulador do processo foram assumidas disponíveis duas medições da variável controlada $h_{w}$ (interface óleo/água), às quais foram adicionados ruídos com média zero e variâncias distintas. Assim, é possível simular duas medições de nível provindas de dois sensores diferentes, com características de medições diferentes e localizadas em pontos diferentes do vaso separador. A implementação e ajustes dos filtros são detalhados em (Oliveira, 2013).

O primeiro teste realizado foi utilizando a primeira técnica do método de fusão de medições. $\mathrm{O}$ gráfico superior da Figura 4 apresenta a estimativa do nível com a fusão de dados aplicada. Visualmente é claro que o resultado obtido é melhor do que qualquer uma das duas medições. A estimativa acompanha as perturbações do sistema e varia muito pouco em relação à saída esperada.
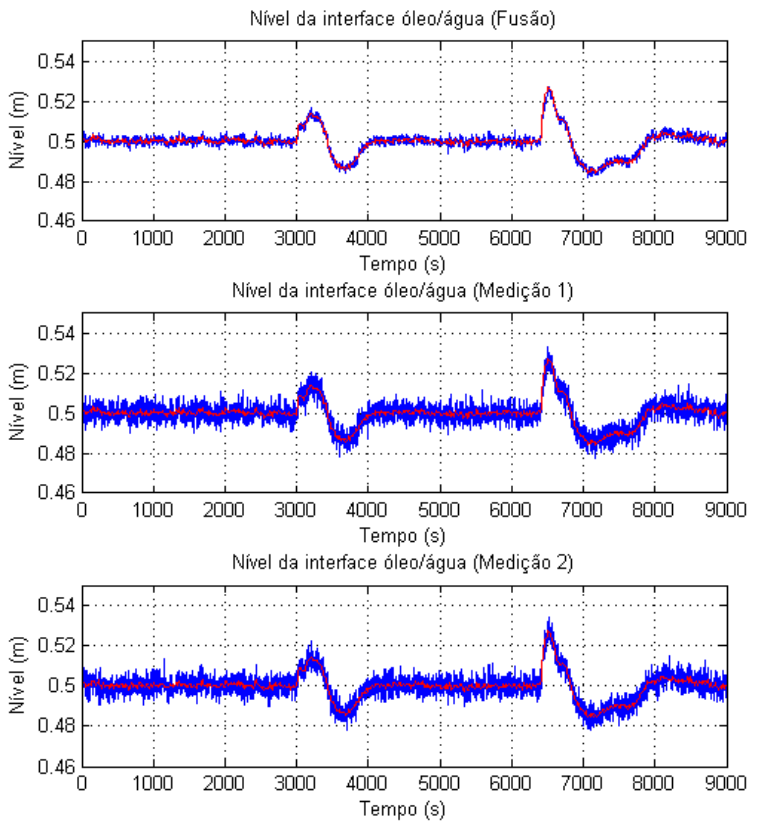

Figura 4. Medições e resultado da aplicação da $1^{\mathrm{a}}$ técnica de fusão de medições.

A análise da Figura 5 indica que a estimativa feita por meio da fusão de dados varia dentro de uma faixa de valores muito pequena. Isto é apenas possível devido às ponderações que são realizadas automaticamente pelo ganho de Kalman dentro do 
algoritmo. Quando o ganho de Kalman possui um valor elevado, o filtro dá maior peso as medições, ou seja, corrige a predição para um valor mais próximo das medições. Quando o valor do ganho de Kalman é baixo, o filtro segue mais de perto as previsões do modelo, suavizando o ruído. Supondo um ganho de Kalman tendendo ao infinito, as previsões do modelo são completamente ignoradas. Caso contrário, quando o ganho de Kalman é igual à zero, as medições são completamente ignoradas.

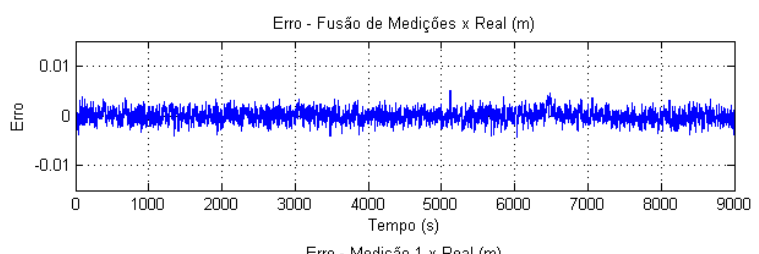

Erro - Medição $1 \times$ Real (m)
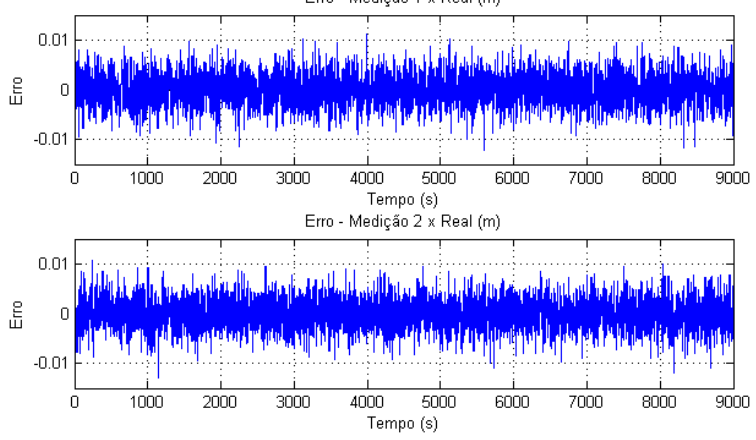

Figura 5. Erro das medições do nível e da estimativa do nível originada pela fusão de dados.

O resultado do cálculo do erro quadrático médio dos três sinais da Figura 5 em relação ao sinal real pode ser visto na Tabela 1 .

Tabela 1. Erro quadrático médio utilizando a $1^{\mathrm{a}}$ técnica de fusão de medições.

\begin{tabular}{|c|c|}
\hline \multicolumn{2}{|c|}{ Erro Quadrático Médio dos Sinais } \\
\hline Fusão das Medições & $0,3308 \times 10^{-5}$ \\
\hline Medição 1 & $1,0261 \times 10^{-5}$ \\
\hline Medição 2 & $0,9844 \times 10^{-5}$ \\
\hline
\end{tabular}

Os dados da Tabela 1 só comprovam ainda mais o que já era esperado. O erro quadrático médio da estimativa feita por meio da fusão das duas medições é muito menor do que o erro quadrático médio de qualquer uma das medições.

Como pode ser visto a aplicação desta técnica de fusão de dados pra estimativa da interface óleo/água mostrou ser bastante eficiente, já que há uma redução do erro de forma significativa, o que traz uma melhor performance do processo de separação primário do óleo bruto.

Para a segunda técnica do método de fusão de medições, foram também realizadas simulações, e da mesma forma que para a técnica anterior, a estimativa da interface óleo/água realizada por meio da fusão das medições se saiu melhor que qualquer uma das medições, com pode ser vista na Figura 6. Isso pelo fato de que o filtro de Kalman trabalha de forma a calcular uma estimativa de variância mínima para um estado, fazendo com que aja uma diminuição do erro quadrático.

$\mathrm{Na}$ Tabela 2, podem ser vistos os valores dos erros quadráticos médios de cada um dos níveis. Da mesma forma que a técnica de fusão de dados da mostrado anteriormente, o resultado da técnica de fusão de medições por ponderação das medições foi melhor do que qualquer uma das medições. Além disso, para esta aplicação, a segunda técnica mostrou superioridade que a técnica anterior, apresentando um menor erro quadrático médio em relação ao valor esperado.

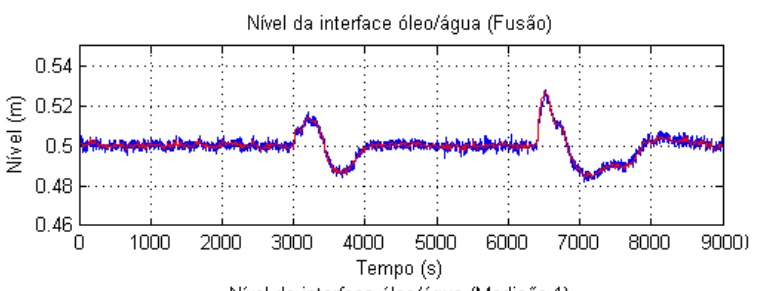

Nivel da interface óleo/água (Medição 1)

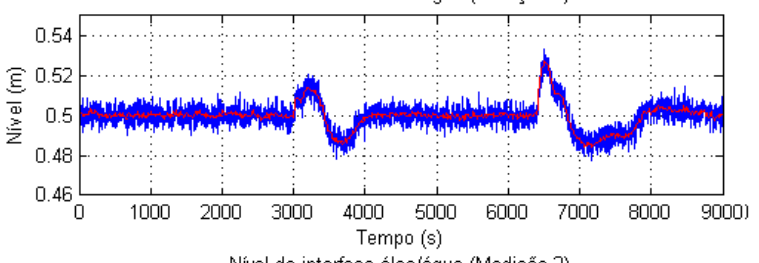

Nivel da interface óleo/água (Medição 2)

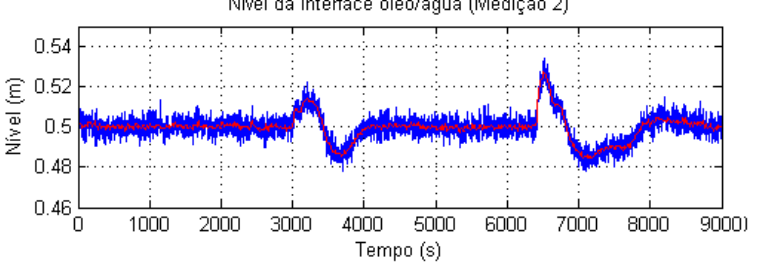

Figura 6. Medições e resultado da aplicação da $2^{a}$ técnica de fusão de medições.

Tabela 2. Erro quadrático médio utilizando a $2^{\mathrm{a}}$ técnica de fusão de medições.

\begin{tabular}{|c|c|}
\hline \multicolumn{2}{|c|}{ Erro Quadrático Médio dos Sinais } \\
\hline Fusão das Mediçõoses & $0,2769 \times 10^{-5}$ \\
\hline Medição 1 & $1,0261 \times 10^{-5}$ \\
\hline Medição 2 & $0,9844 \times 10^{-5}$ \\
\hline
\end{tabular}

O segundo método de fusão de dados é um pouco diferente. Ao contrário das técnicas anteriores, que primeiramente fazem a fusão das medições para, em seguida, efetuar estimação do nível da interface óleo/água, este método faz a estimação do nível utilizando cada uma das medições e, em seguida, estas estimativas são fundidas em uma só.

$\mathrm{Na}$ Figura 7, podem ser vistos os resultados dos níveis das medições individuais dos sensores e do nível estimado a partir da fusão das estimativas de nível por meio de cada uma das medições individuais.

Como já era esperado, o resultado do valor estimado é muito melhor que qualquer uma das medições individuais. Coforme dito, o filtro de Kalman é um dos melhores métodos para filtragem 
estocástica e, associado à fusão de dados, torna-se uma grande ferramenta para contornar erros aleatórios dos quais os processos estão susceptíveis.

Verificando na Tabela 3 os valores dos erros quadráticos médios dos níveis em relação ao valor esperado, fica nítido o potencial desse método. $\mathrm{O}$ valor do erro do nível proveniente da fusão das estimativas se mostrou inferior às técnicas anteriores.

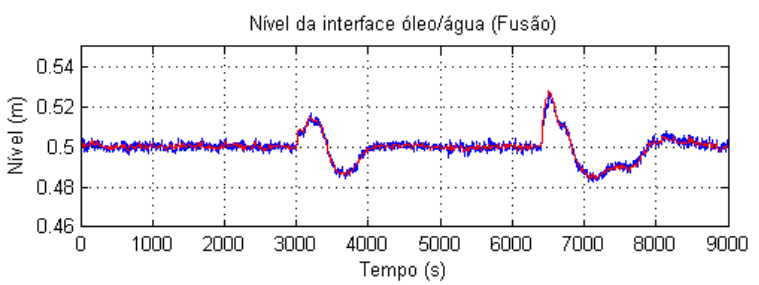

Nivel da interface óleo/água (Medilçấo 1)

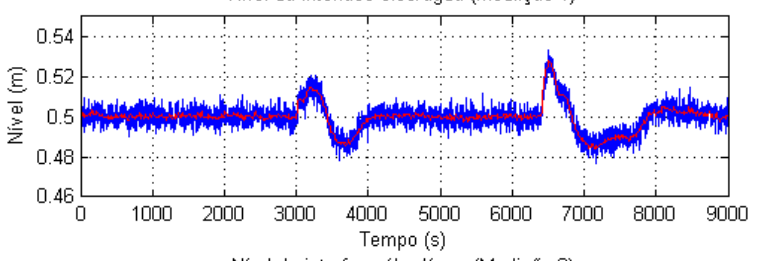

Nivel da interface óleo/água (Mediçẫo 2)

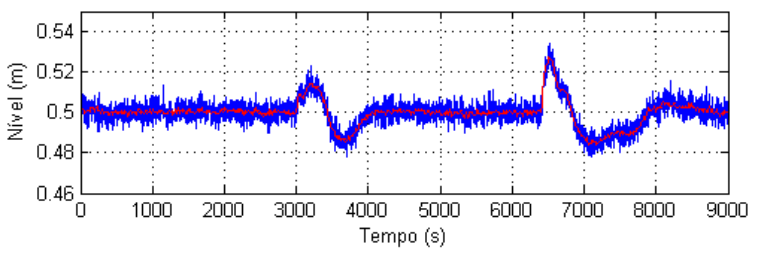

Figura 7. Medições e resultado da aplicação do método de fusão de estimativas.

Tabela 3. Erro quadrático médio para fusão de estimativas.

\begin{tabular}{|c|c|}
\hline \multicolumn{2}{|c|}{ Erro Quadrático Médio dos Sinais } \\
\hline Fusão das Estimativas & $0,2268 \times 10^{-5}$ \\
\hline Medição 1 & $1,0261 \times 10^{-5}$ \\
\hline Medição 2 & $0,9844 \times 10^{-5}$ \\
\hline
\end{tabular}

\section{Conclusões}

Este trabalho teve como objetivo implementar um algoritmo capaz de gerar uma estimação on-line dos nível da interface óleo/água num processo de separação, por meio da utilização de filtros de Kalman e fusão de dados, a fim de melhorar a detecção, certeza e confiabilidade dos dados no controle do processo.

Os diversos métodos apresentados acerca da utilização do filtro de Kalman para fusão de dados funcionaram como o esperado. A técnica se mostrou bastante eficiênte para estimativa de medições. Os valores obtidos foram, de longe, mais próximos aos valores esperados do que qualquer uma das medições dos sensores físicos simulados. Assim, faz com que aja um controle mais eficiênte da planta, gerando um melhor desempenho no sistema de separação de petróleo.

Por meio das simulações realizadas, pôde-se perceber a superioridade do método de fusão de estimativas. $\mathrm{O}$ erro quadrático médio gerado por este método conseguiu ser menor que os anteriores. Logo, a sua utilização é a mais promissora para servir como entrada de realimentação o controlador do sistema, proporcionando um controle mais eficiente da planta.

\section{Agradecimentos}

Os autores agradecem o auxílio financeiro do projeto UFS-UFRN-Petrobras “AIPetro".

\section{Referências Bibliográficas}

Figueiredo, A. (2009). Detecção, diagnóstico $e$ correção de situações anormais em processos de produção de petróleo. Relatório $\mathrm{PIBIC/CNPq}$ 726, Universidade Federal de Sergipe, Brasil.

Filho, M. B. C.; Schilling, M. T.; Souza, J. C. S. (2007). Sobre o problema da integração generalizada de dados. Revista Controle \& Automação, 18(1): 24-43. DOI: 10.1590/S0103-17592007000100003

Gao, J. B.; Harris, C. J. (2002). Some remarks on Kalman filters for the multisensor fusion. Information Fusion, 3(3), 191-201. DOI: 10.1016/S1566-2535(02)00070-2

Harris, C.; Bailey, A.; Dodd, T. (1988). Multisensor data fusion in defense and aerospace. The Aeronautical Journal, 102(1015): 229-244.

Kalman, R. E. (1960). A new approach to linear filtering and prediction problems. Transactions of the ASME, Journal of Basic Engineering, 82(1):35-45. DOI: 10.1115/1.3662552

Khaleghi, B.; Khamis, A.; Fakhreddine, O.K.; Saiedeh, N. R. (2013). Multisensor data fusion: A review of the state-of-the-art. Information Fusion 14(1), 28-44. DOI: 10.1016/j.inffus.2011.08.001

Nazar, M. S. M. (2009). A comparative study of differente Kalman filtering methods in multi sensor data fusion. In: Proceedings of the Int. Multi-Conference of Engineers and Computer Scientists (IMECS 2009), Hong Kong. China.

Oliveira, L.B. (2013). Fusão de dados baseada no filtro de Kalman para estimação do nível da interface óleo/água em processos de separação. Trabalho de Conclusão de Curso em Engenharia Elétrica, Universidade Federal de Sergipe, Brasil.

Santos, R.R.; Sotomayor, O.A.Z. (2012) MPC com controle das saídas por faixas de um processo de produção de petróleo "offshore". In: Anais do XIX Congresso Brasileiro de Automática (CBA 2012), Campina Grande-PB.

Silveira, M.A.C.R. (2006). Controle de um processo de tratamento primário de petróleo. Dissertação de Mestrado, COPPE/Universidade Federal do Rio de Janeiro, Rio de Janeiro-RJ, Brasil.

Willner, D.; Chang, C.; Dunn, K. (1976). Kalman filter algorithms for a multi-sensor system. In: Proc. of the IEEE Conference on Decision and Control (CDC 1997), Clearwater, FL. 